\title{
Drenaje pluvial sostenible. Una alternativa de gestión del agua de lluvia en la Universidad de Sonora.
}

\author{
Sustainable stormwater drainage. A rain water management alternative at the \\ University of Sonora.
}

Recibido: enero 2019

Aceptado: marzo 2020

\section{Resumen}

En este artículo se presenta un estudio que desarrolló con motivo del recurrente problema de inundación que se presenta en la zona urbana de Hermosillo en temporada de lluvias, y en el principal campus de la Universidad de Sonora. El estudio consistió en la evaluación del sistema de drenaje pluvial en el campus universitario mediante la aplicación de herramientas y principios hidrológicos, hidráulicos, topográficos y sociales para determinar los caudales que genera el escurrimiento del agua pluvial en las doce microcuencas identificadas, derivado del proceso estadístico de las lluvias máximas diarias de una serie de anual de 34 años de registro climático en la estación meteorológica del centro de la ciudad. Se obtuvieron las curvas de precipitación-duración-periodo de retorno (P-d-Tr) eligiendo una tormenta de diseño para un periodo de retorno de 10 años, y una duración de la tormenta igual al tiempo de concentración que fue variable para cada una de la microcuencas. Una vez definidos los caudales se calcularon los elementos hidráulicos necesarios para captar y conducir el agua pluvial; finalmente se estableció la modalidad para aprovechar el agua en la gestión del agua de lluvia atendiendo un aprovechamiento sostenible.

\section{Palabras Clave:}

precipitación pluvial; microcuenca; drenaje sostenible

\author{
Arturo Ojeda de la Cruz ${ }^{1}$ \\ Clara Rosalía Álvarez Chávez ${ }^{2}$ \\ David Carlos Orona Llano ${ }^{3}$
}

\begin{abstract}
This article presents a study that was developed due to the recurring problem of flooding that occurs in the urban area of Hermosillo during the rainy season, and on the main campus of the University of Sonora. The study consisted of the evaluation of the pluvial drainage system in the university campus through the application of hydrological, hydraulic, topographic and social tools and principles to determine the flows generated by the runoff of rainwater in the twelve micro-basins identified, derived from the statistical process of the maximum daily rainfall of a series of 34-year annual climate record at the meteorological station of the city center. The precipitation-duration-return period (P-d-Tr) curves were obtained by choosing a design storm for a period of 10 years, and a duration of the storm equal to the concentration time that was variable for each of the micro-watersheds Once the flows were defined, the hydraulic elements necessary to capture and conduct the rainwater were calculated; Finally, the modality to take advantage of water in rainwater management was established, taking into account sustainable use.
\end{abstract}

\section{Keywords:}

rainfall; microwatershed; drainage sustainable

\footnotetext{
1 Nacionalidad: mexicano; adscripción: Departamento de Ingeniería Civil y Minas / División de Ingeniería, Universidad de Sonora; Doctor en Filosofía con orientación en Asuntos Urbanos; miembro del Sistema Nacional de Investigadores CONACYT y líder del Cuerpo Académico Gestión Urbana reconocido en el nivel en consolidación; E-mail: ojeda@dicym.uson.mx

2 Nacionalidad: mexicana; adscripción: Profesora adjunta del Departamento de Salud Pública de la Universidad de Massachusetts-Lowell; doctorado en Ciencias con área de concentración en producción más limpia y prevención de la contaminación (University of MassachussettsLowell). Es integrante del Sistema Nacional de Investigadores, Nivel I del CONACYT; E-mail: pissa_unison@hotmail.com

3 Nacionalidad: mexicano; adscripción departamento de Ingeniería Industrial. División de Ingeniería; egresado del posgrado Ciencias de la Sustentabilidad; E-mail: david.oronallano@hotmail.com
} 


\section{INTRODUCCIÓN.}

La combinación del cambio climático y la urbanización está empeorando los problemas de inundación en zonas urbanas; estimar la cantidad de lluvia que una ciudad puede tolerar sin inundar es una tarea fundamental, aunque se invierten grandes cantidades de recursos en el control de inundaciones urbanas (Chi-Feng and ChungMing, 2014). Con el cambio climático global y la rápida urbanización, el volumen de escurrimiento de aguas pluviales y la máxima descarga se han incrementado considerablemente durante la temporada de lluvias debido a la mayor frecuencia y alta intensidad de los eventos de tormentas extremas y el rápido incremento de la superficie impermeable en las zonas urbanas (Sun et al. 2014). Esto ha estado causando un aumento de los riesgos de inundación en las areas bajas de una ciudad, causando importantes problemas sociales y pérdidas económicas (Li Fei et al., 2015).

Las ciudades de todo el mundo enfrentan múltiples desafíos, incluida la expansión de las áreas pavimentadas, la pérdida de la cubierta vegetal y los efectos del cambio climático. Los sistemas convencionales de drenaje son particularmente afectados ya que normalmente su diseño inicial se ha basado en transportar rápidamente el agua de lluvia hacia las aguas receptoras; con frecuencia su capacidad está comprometida por el aumento de las áreas impermeables que producen grandes cantidades de escorrentía que se espera aumenten aún mas en muchas partes del mundo debido al cambio climático (Perales-Momparler, 2016; Brown et al., 2009). Lo cual provoca que las ciudades sean cada vez más vulnerables a las inundaciones debido a la rápida urbanización, Instalación de infraestructura compleja convencional, y cambios en los patrones de precipitación causada por el cambio climático antropogénico (Willems et al., 2012).

En este aspecto, las autoridades locales pueden carecer de conocimiento y de la experiencia de los procesos de aplicación de planificación en lo general, y específicamente en lo referente al sistema de drenaje sostenible; siendo un área clave lo referente a los controles en la construcción de dicho tipo de drenaje, así como de su mantenimiento (Bryan and Lundy, 2016). Está bien establecido que la práctica tradicional de la gestión de las aguas pluviales urbanas contribuye a la degradación de la recepción de las vías fluviales, y esta práctica ha sido criticada por facilitar el despilfarro de un valioso recurso hídrico. Sin embargo, a pesar de los significativos avances en las técnicas y procesos alternativos de "gestión integrada de aguas pluviales urbanas" durante los últimos 20 años, la implementación a gran escala ha sido limitada. Este problema es un indicio de impedimentos institucionales más amplios que van más allá de las preocupaciones actuales de fortalecer la experiencia en procesos tecnológicos y de planificación (Brown R., 2005).

La urbanización es una tendencia global con efectos adversos sobre los recursos hídricos, ya que el aumento dramático de la superficie impermeable genera problemas de inseguridad al incrementarse el escurrimiento de agua siendo de mayor riesgo las zonas de menor altitud, disminuye la recarga de agua a los acuíferos, entre otros (Katsifarakis et al., 2015). La gestión el agua de lluvia es un proceso que busca mejores prácticas del uso del suelo del paisaje construido, lo cual incluye la prevención de la escorrentía adecuando areas para detener la escorrentía de agua y reducir su flujo hacia los arroyos, o bien, adecuando areas con vegetación para la provocar filtración del agua (RCL, 2004). En el caso del aprovechamiento del agua, las principales técnicas utilizadas contemplan el uso de estructuras hidráulicas que buscan reproducir la capacidad de infiltración de agua en el subsuelo, creando como resultado un volumen de agua más pequeño de escurrimiento superficial, reduciendo con ello los problemas de inundación; además, esto promueve la recarga de acuíferos y mejoras en la calidad del agua (Poleto and Tassi, 2012). Esto es en virtud de que, los sistemas de drenaje convencionales en zonas altamente urbanizadas son incapaces de controlar tanto la cantidad de escorrentía como la calidad del agua; en consecuencia, los sistemas convencionales de drenaje han demostrado ser una opción insostenible que impacta tanto en los ambientes terrestres como acuáticos; por lo que pueden utilizarse canales de conducción de agua y zanjas filtrantes para aprovechar el agua pluvial hacia el subsuelo (Sharma and Kansal, 2013). Al buscar adaptarse a estas nuevas exigencias, se hace necesario un nuevo enfoque para la gestión de las escorrentías, lo cual ha llevado al interés creciente por el uso de Sistemas Urbanos de Drenaje Sostenible (SUDS); estos comprenden un amplio espectro de soluciones que permiten afrontar el planeamiento, diseño y gestión de las aguas pluviales dando tanta importancia a los 
aspectos medioambientales y sociales como a los hidrológicos e hidráulicos (Perales-Momparler y Doménech, s.f.).

En ese sentido, los SUDS, son definidos como elementos integrantes de la infraestructura hidráulica urbana, promovientes del paisaje (naturalizados), y destinados a filtrar, retener, infiltrar, transportar y almacenar agua de lluvia, de forma que ésta no sufra ningún deterioro o incluso permita la eliminación, de forma natural, de parte de la carga contaminante que haya podido adquirir por procesos de escorrentía urbana previa (Benavides y Perales-Momparler, 2008). De ahí que, las transiciones hacia entornos urbanos construidos regenerativos que incorporan ampliamente sistemas de drenaje sostenible son aspectos innovadores, pero que encuentran barreras sobre la evidencia del desempeño de estos sistemas que, en muchos países todavía son desconocidos por los profesionales y los tomadores de decisiones (Perales-Momparler et al., 2016). Estas herramientas de diseño urbano sensible al agua, están diseñadas para almacenar, infiltrar y captar el agua en la fuente, fomentando la evaporación, evapotranspiración, recarga de aguas subterráneas y reutilización de aguas pluviales. Si bien hay numerosas demostraciones de prácticas WSUD, hay pocos ejemplos de implementación generalizada a escala de cuencas (Roy, et al., 2008). Esta recuperación de agua pluvial se denomina también técnicas compensatorias de drenaje urbano (TCs), poseen el concepto de resolver el problema del drenaje de aguas pluviales en la fuente, buscando compensar los efectos de la urbanización a través de la retención o almacenaje de agua por la infiltración. La búsqueda de soluciones alternativas y sustentables viene aumentando la utilización de TCs, algunos ejemplos son estanques de detención, áreas de infiltración, pozos de infiltración, zanjas, trincheras (Gonçalves y otros, 2016).

El objetivo del presente artículo es mostrar un estudio en el que se estableció un sistema de drenaje pluvial con enfoque sostenible en el campus universitario más importante de la Universidad de Sonora, localizado en la ciudad de Hermosillo. Se colecta el agua pluvial que escurre superficialmente e ingresa al campus por la misma situación de escorrentía urbana. Esto es en virtud de que continuamente al presentarse tormentas ligeras y/o abundantes genera un caos al interior del campus ya que los estudiantes quedan prácticamente incomunicados al no poder desplazarse entre las calles y edificios; además del conflicto vehicular que se genera por la acumulación de agua. La alternativa de solución propuesta contribuye al conocimiento sobre los sistemas urbanos de drenaje sostenible, permitiendo proponer un sistema propio para el sitio en cuestión que puede ser referente para otros campus universitarios.

\section{MATERIALES Y MÉTODOS.}

\subsection{DESCRIPCIÓN DE LA ZONA DE ESTUDIO.}

El Estado de Sonora se localiza al noroeste de México en la zona más árida del territorio nacional, conocida como Desierto de Sonora que se extiende al sur de Estados Unidos de América a través de los estados de Arizona y California, y en México por el Estado de Baja California y Sonora. La ciudad de Hermosillo, Sonora se ubica en la parte central-poniente de la planicie costera del estado, se dispone en la región noroeste de México (fig.1) a una elevación de 210 metros sobre el nivel del mar (msnm), y se localiza geográficamente a los $29^{\circ} 06^{\prime}$ de latitud norte y $110^{\circ} 58^{\prime}$ de longitud oeste (Ojeda et al., 2017).

Esta ciudad capital concentra el $27 \%$ de la población estatal $(2,662,480$ habitantes $)$ de acuerdo a los datos censales del año 2010 del Instituto Nacional de Estadística Geografía e Informática (INEGI). El clima cálido desértico se siente prácticamente durante cinco meses del año predominando una temperatura máxima de 42 grados centígrados y un valor extremo de $46{ }^{\circ} \mathrm{C}$ $\left(108^{\circ} \mathrm{F}\right.$ y $\left.115^{\circ} \mathrm{F}\right)$ (CONAGUA, 2016).

Por otro lado, la Universidad de Sonora se conforma en por unidades regionales con seis campus. La unidad regional centro (fig. 2) tiene una superficie de 70 hectáreas, en donde se alberga el campus principal de la Unidad Regional Centro denominado campus Hermosillo, sitio donde se localiza el area de estudio. La Universidad dispone en global de 4,785 empleados en de todos los sectores, de los cuales prácticamente el $80 \%$ se concentra en la unidad centro, campus Hermosillo. En los espacios peatonales, estacionamientos, esparcimiento y areas académicas se tiene una movilidad de 14,500 personas (incluyendo estudiantes) que se encuentran en tránsito peatonal diariamente al interior del campus en cuestión; y en actividades diversas; además de que el flujo vehicular es alto ya que circulan en promedio 20000 automóviles cada día. Las vialidades principales que son paralelas denominadas 
Universidad, y Sonora, son las calles más transitadas diariamente en el campus (PDI, 2013). Estas dos avenidas mencionadas conducen el mayor caudal de agua $\left(\mathrm{m}^{3} / \mathrm{seg}\right)$ producto del escurrimiento de agua pluvial que ingresa al campus y del sitio mismo.

Figura 1. Localización geográfica de la zona de estudio

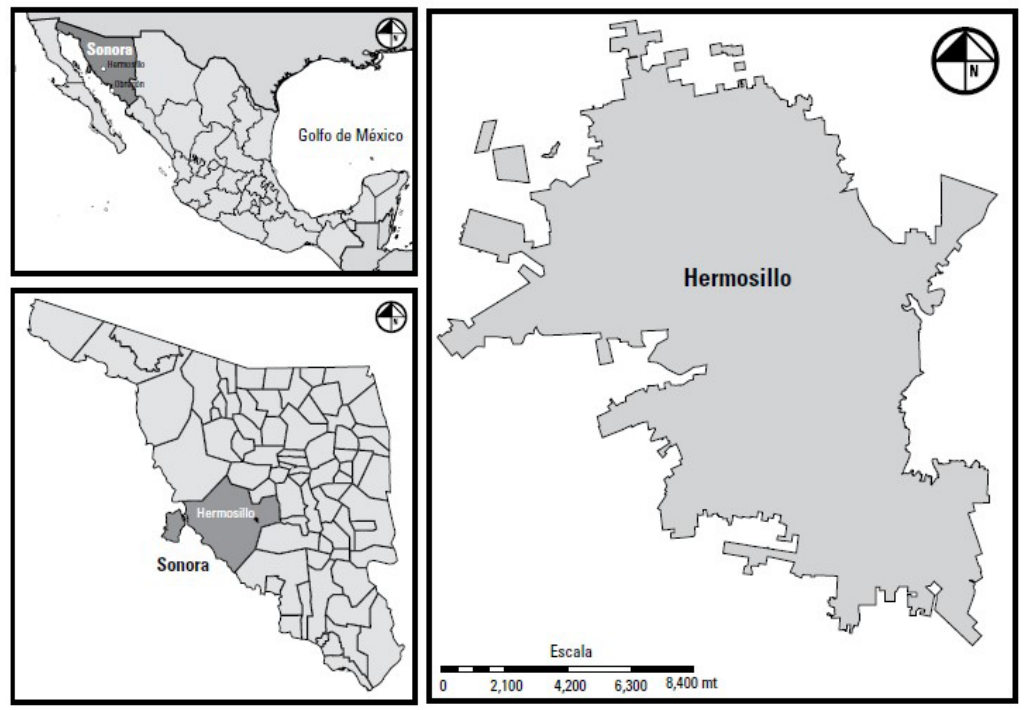

Figura 2. Campus Hermosillo, Universidad de Sonora

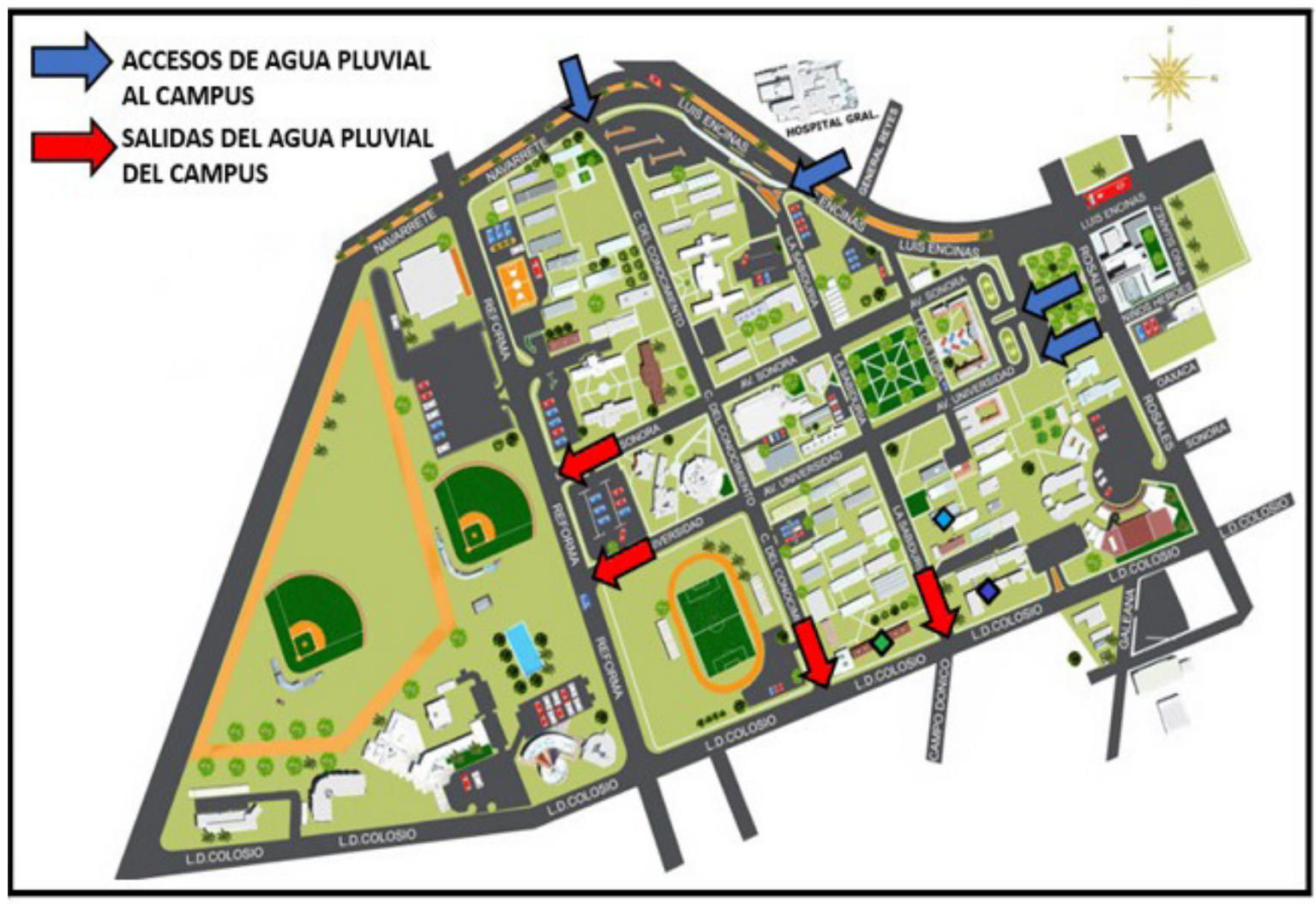

Fuente 1: Ojeda, Narváez y Quintana (2014); Fuente 2: elaboración propia 


\subsection{DISEÑO DEL ESTUDIO.}

El alcance del estudio es para una sección del campus donde se presenta la mayor inundación de vialidades y que abarca 40 hectáreas de superficie, comprendido entre los límites de las vialidades urbanas Ave. Reforma - Blvd. Navarrete - Blvd. Luis Encinas - Ave. Rosales - Ave. Colosio, lo cual distingue en la sección oriente del mapa de la figura 2. El estudio se realizó partiendo de la colección de datos históricos de la precipitación pluvial máxima diaria ocurrida en la estación climatológica más cercana al sitio de estudio, siendo ésta la "estación meteorológica Observatorio de Hermosillo" perteneciente a la Comisión Nacional del Agua (CONAGUA).

Para lograr el objetivo planteado se requirió definir y establecer lo siguiente:

1. Colectar información de las precipitaciones pluviales históricas en la estación climatológica del centro de la ciudad proporcionado por CONAGUA.

2. Se identificó la subcuenca urbana general y las microcuencas que influyen. Delimitando cada una a través de los parteaguas correspondientes; obteniendo sus características básicas para el análisis. identificando mediante planos $\mathrm{e}$ información topográfica la ruta del escurrimiento del agua al interior del campus universitario.
3. Se procesó estadísticamente la serie de datos para obtener las precipitaciones máximas, sus duraciones y periodos de retorno. obteniendo la tormenta de diseño.

4. Determinación de la tormenta y caudal de diseño, así como las propuestas de la red de conducción del agua, para lograr el diseño de la red e integración del modelo de Drenaje Pluvial Sustentable (DPS).

Una cuenca es el área limitada por un parteaguas que drena el agua recibida y la dirige hacia el sitio de salida que corresponde siempre al punto topográfico más bajo del área de estudio. Esta etapa del estudio fue básica, puesto que correspondió a visualizar la cuenca urbana que influye en el sitio de estudio, y a su vez identificar las microcuencas que participan con el escurrimiento de agua hacia el campus universitario. Para ello, se revisó la información de los niveles topográficos en el área perimetral y mediante recorrido de campo al interior del campus, identificándose las microcuencas (fig. 3). En dicha figura cada microcuenca se delimitó con un tono de color distinto, para diferenciar el area de escurrimiento, y a su vez resaltar tres microcuencas externas (Ce) que tienen su mayor parte de superficie fuera del campus universitario, mientras que también se identificaron nueve subcuencas internas $(\mathrm{Ci})$.

Figura 3. Microcuencas de influencia hacia y en el campus universitario

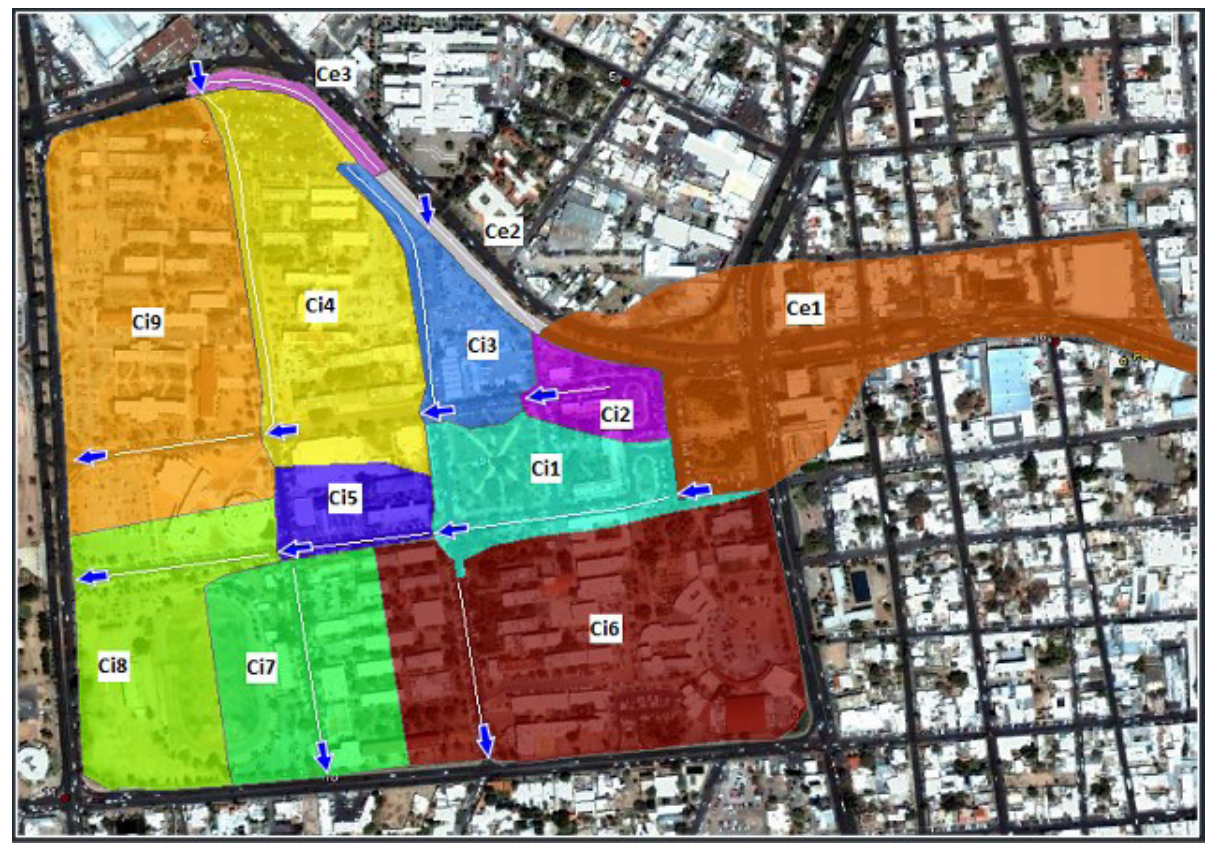

Fuente: elaboración propia 


\subsection{PROCESO ESTADÍSTICO.}

La información colectada de los eventos de precipitación máxima diaria abarcó el periodo anual desde 1982 hasta el año 2015, conformando una serie anual de 34 años de registro pluvial de los eventos extremos en la estación meteorológica Observatorio (Tabla 1), operada por la CONAGUA. Destaca aquí que el valor más bajo registrado fue de $23 \mathrm{~mm}$ ocurrido en agosto del 2009, y los dos valores más altos del registro base fueron 109.8 mm y 103 mm en septiembre del año 1988 y 2015 respectivamente.

El periodo de retorno o intervalo de recurrencia se define como el número de años que en promedio se presenta un evento de una intensidad determinada, obtenido éste con el inverso de la probabilidad de ocurrencia (CONAGUA, 2007). Mientras que el cálculo de las curvas Precipitación-duraciónPeriodo de retorno (P-d-Tr) se basa en el procesamiento estadístico de las lluvias máximas diarias disponibles para lograr así obtener los valores probables de lluvia en 24 horas de los periodos de retorno elegidos para construir las curvas $\mathrm{P}-\mathrm{d}-\mathrm{Tr}$. Cuando el número de años del registro de las lluvias en la estación meteorológica de estudio es mayor o igual que los periodos de retorno para los que se requieren obtener las lluvias máximas en 24 horas, entonces debe utilizarse en el proceso estadístico el criterio de interpolación; en cuyo caso, los valores buscados de las precipitaciones se deducen a partir de una ecuación de regresión lineal entre los valores de los eventos de la serie anual, como se muestra en la ecuación (1), (Campos-Aranda, 1998).

$$
P_{T r}=A+B(\log T r)
$$

Donde:

$P_{T r}=$ Precipitación máxima diaria del periodo de retorno $\mathrm{Tr}$, en milímetros.

A, B = Parámetros de ajuste de la regresión lineal. $\operatorname{Tr}=$ Periodo de retorno de la serie anual de máximos, en años. Obtenido a través de la Ec. 2 de Weibull

$$
\operatorname{Tr}=\frac{n+1}{m}
$$

Siendo:

$\mathrm{n}=$ número de años de registro en la estación meteorológica en cuestión.

$\mathrm{m}=$ número de orden del evento, arreglados los datos en orden decreciente.
Tabla 1. Registro de lluvia máxima diaria

\begin{tabular}{|c|l|c|}
\hline Año & \multicolumn{1}{|c|}{ Mes } & P Max $(\mathbf{m m})$ \\
\hline 1982 & Diciembre & 67.6 \\
\hline 1983 & Julio & 41.7 \\
\hline 1984 & Agosto & 63.1 \\
\hline 1985 & Agosto & 76.3 \\
\hline 1986 & Julio & 106.7 \\
\hline 1987 & Agosto & 32.6 \\
\hline 1988 & Septiembre & 109.8 \\
\hline 1989 & Septiembre & 100.0 \\
\hline 1990 & Agosto & 102.5 \\
\hline 1991 & Diciembre & 32.7 \\
\hline 1992 & Agosto & 121.7 \\
\hline 1993 & Abril & 48.2 \\
\hline 1994 & Diciembre & 79.1 \\
\hline 1995 & Febrero & 61.5 \\
\hline 1996 & Agosto & 54.2 \\
\hline 1997 & Julio & 34.0 \\
\hline 1998 & Agosto & 22.8 \\
\hline 1999 & Agosto & 50.1 \\
\hline 2000 & Octubre & 74.3 \\
\hline 2001 & Octubre & 83.2 \\
\hline 2002 & Noviembre & 45.1 \\
\hline 2003 & Agosto & 40.4 \\
\hline 2004 & Julio & 30.0 \\
\hline 2005 & Febrero & 30.3 \\
\hline 2006 & Octubre & 52.9 \\
\hline 2007 & Julio & 63.4 \\
\hline 2008 & Agosto & 28.0 \\
\hline 2009 & Agosto & 23.0 \\
\hline 2010 & Julio & 92.1 \\
\hline 2011 & Noviembre & 65.9 \\
\hline 2012 & Agosto & 71.8 \\
\hline 2013 & Septiembre & 41.3 \\
\hline 2014 & Septiembre & 33.9 \\
\hline 2015 & Septiembre & 103.9 \\
\hline
\end{tabular}


Obtenidos los parámetros de ajuste A y B, y dando forma a la ecuación, se determinaron los valores de las lluvias máximas diarias para cada los periodos de retorno elegidos: 2, 5, 10, 20, 25 y 50 años. Dichas precipitaciones se corrigieron por el factor (F) de intervalo fijo de observación utilizando un incremento $\mathrm{F}=1.13$ de acuerdo al criterio sugerido en ello, y por Campos (1998). Esto permitió considerar como valores definitivos de lluvias máximas en 24 horas para la estación meteorológica en cuestión, y en consecuencia para el sitio de estudio como valor general en cada periodo de retorno, pero sin considerar aún la duración de la tormenta, lo cual es un proceso complementario para obtener las curvas P-d-Tr. El cálculo de los parámetros de ajuste A y $B$ de la ecuación (1) se determinaron de acuerdo a Campos-Aranda (1998) quien describe ampliamente el proceso.

En ese sentido, F.C. Bell citado por CamposAranda (1998) logró tomar resultados de numerosas investigaciones referente a este proceso y obtener la lluvia de duración de una hora y periodo de retorno de 2 años $\left(P_{2}^{60}\right)$, ecuación (3). Resultando finalmente el valor para el presente estudio de $32.6 \mathrm{~mm}$, y aplicando los criterios definidos en Campos (1998); mismo que presenta la ecuación de las lluvias máximas en 24 horas para duraciones distintas (en minutos) de la tormenta y sus respectivos periodos de retorno, esto es:

$P_{T r}^{t}=(0.35 \operatorname{Ln} \operatorname{Tr}+0.76)\left(0.54 t^{0.25}-0.50\right) P_{2}^{60}$

Siendo:

$P_{T r}^{t}=$ Precipitación $(\mathrm{mm})$ de duración $t$ minutos y periodo de retorno $\mathrm{Tr}$ en años.

$P_{2}^{60}=$ Precipitación de duración una hora y periodo de retorno 2 años. Campos-Aranda (1998) menciona y describe que debe ser estimado conforme a los criterios de U.S. Weather Bureau, y B.M. Reich, orientado y establecido en su libro proceso del ciclo hidrológico.

Finalmente, las duraciones de tormenta (minutos) sugeridas para obtener y presentar las curvas precipitación, duración y periodo de retorno $\mathrm{P}-\mathrm{d}-\mathrm{Tr}$, fueron los valores siguientes: 5, 10, 20, 40, 60, 100, 120 , y 1440 minutos o $24 \mathrm{hrs}$, mismos que se tomaron en cuenta en el procesamiento para determinar las curvas P-d-Tr del presente estudio (Fig. 4).

\section{DISEÑO DEL SISTEMA.}

\subsubsection{GASTO MÁXIMO EN LA CUENCA.}

El método Racional es el modelo más antiguo de la relación lluvia-escurrimiento y debido a su sencillez es uno de los más utilizados; se basa en considerar que, sobre el área estudiada se tiene una lluvia uniforme durante un cierto tiempo, de manera que el escurrimiento en la cuenca se establezca y se tenga un caudal constante en la descarga. Este método permite determinar el gasto máximo provocado por una tormenta suponiendo que esto se alcanza cuando la intensidad de lluvia es aproximadamente constante durante una cierta duración, la cual se considera es igual al tiempo de concentración de la cuenca (CONAGUA, 2007). El método racional sólo es aplicable para cuencas pequeñas en donde las variaciones espaciales de la lluvia son reducidas (Campos-Aranda, 2010). La ecuación por este método en la presentación de unidades del sistema métrico, se plantea como:

$$
\mathrm{Q}=0.2778 \mathrm{C} \text { i A }
$$

Donde:

$\mathrm{Q}$ = gasto o caudal máximo en la descarga de la cuenca, $\left(\mathrm{m}^{3} / \mathrm{seg}\right)$

$\mathrm{C}=$ coeficiente de escurrimiento (adimensional, en fracción decimal)

$\mathrm{A}=$ área de la cuenca $\left(\mathrm{Km}^{2}\right)$

$\mathrm{i}=$ intensidad media de la lluvia para una duración de tormenta igual al tiempo de concentración (Tc) de la cuenca $(\mathrm{mm} / \mathrm{hr})$

El tiempo de concentración $(\mathrm{Tc})$ se define como el tiempo que tarda una gota de agua en viajar desde el punto más alejado de la cuenca hasta la salida considerada de esta. Para el presente estudio, el valor de Tc fue estimado con dos criterios, uno fue el criterio del Soil Conservation Service (SCS) y el de la Agencia Federal de Aviación (AFA), adoptando finalmente el promedio de los valores obtenidos en cada microcuenca. La duración de la tormenta como Tc fue calculada para cada una de las microcuencas presentadas anteriormente en la Figura 3.

Mientras que el coeficiente de escurrimiento (C) podría tomar valores extremos de 0 a 1 , ya que está definido por la relación del volumen de agua llovido entre el volumen de agua escurrido en la cuenca respectiva; lo cual estará en función 
de su tipo de suelo y cobertura. Existe una amplia bibliografía sobre los valores posibles que puede adoptar el coeficiente de escurrimiento dependiendo de las características físicas de la cuenca, por ejemplo, un tipo de suelo con cobertura muy impermeable, o bien cuando está completamente mojado y saturado puede producir casi el $100 \%$ de escurrimiento del agua pluvial que recibe. Los valores de dicho coeficiente se establecen en CONAGUA (2007).

Del rango de valores ya establecidos para este coeficiente debe ponerse especial atención cuando la cuenca tiene diversos tipos de suelo y cobertura (suelo natural, jardines, edificios, asfalto, etc.), en este caso se determinó un valor para cada segmento de cuenca y su valor final fue ponderado para lograr un valor de dicho coeficiente de escurrimiento (C) tomando en cuenta el área particular de las secciones de cuenca y el área total de la misma. La intensidad de la lluvia para la duración en cada una de las microcuencas y la precipitación que le corresponde a cada microcuenca se obtuvo apoyándose en las curvas P-d-Tr.

Figura 4. Escurrimiento de agua en el campus por la lluvia del 7 sept. 2016
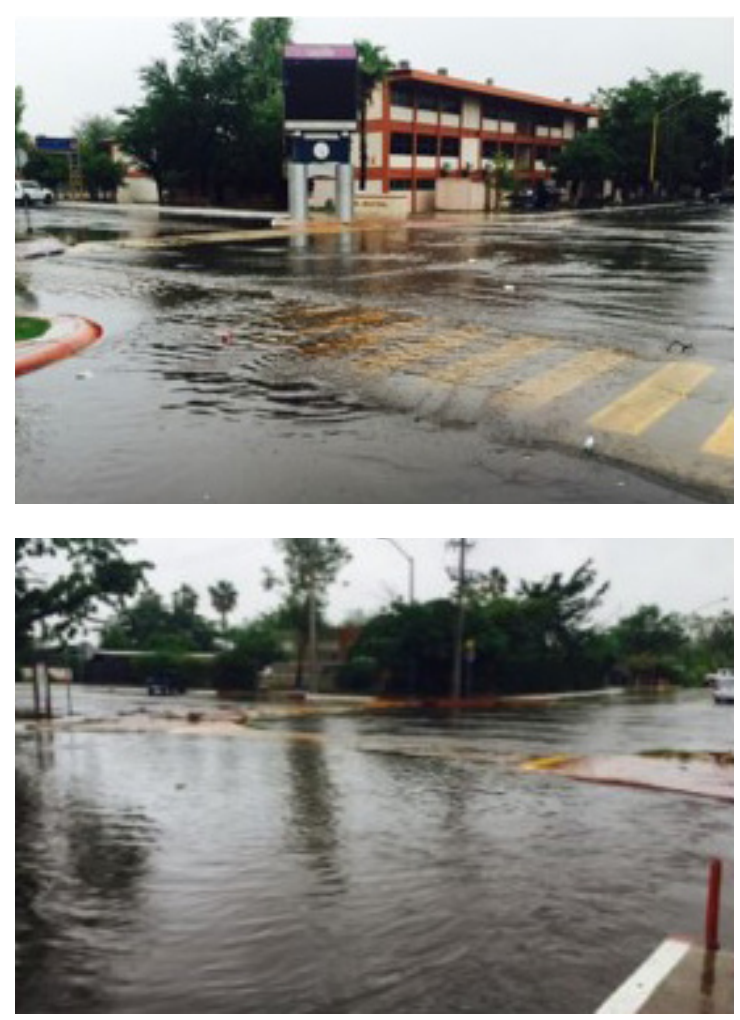

Fuente: elaboración propia
El periodo de retorno que se estableció para elegir dicha precipitación y determinar la intensidad de lluvia para una duración de tormenta, y calcular los gastos (Q) del método racional, fue elegido finalmente el valor de 10 años, el cual es válido para el caso de la opción de drenaje urbano (CONAGUA, 2007).

\subsubsection{SISTEMA DE CONDUCCIÓN DEL AGUA.}

El sistema de conducción del agua pluvial previsto en el presente estudio se conformó en tres etapas básicas: la primera se refiere a la planeación y definición de la representación del sistema general de conducción del agua, esto permitió la identificación de los elementos principales para proceder al diseño de la red de tuberías del alcantarillado pluvial y la captación del agua escurrida. Una segunda etapa se enfocó a identificar los puntos críticos a nivel manzana para proponer una serie de cisternas de captación de agua pluvial (almacenamiento) para su futuro reúso y evitar la sobresaturación del área de jardines, socavaciones y pérdida del agua pluvial. La tercera etapa consistió en completar el ciclo de conducción a superficie libre mediante un canal de tierra de sección trapecial, y a su vez el proceso de infiltración del agua hacia el subsuelo al mismo tiempo que se conduce el agua a través del mismo canal de tierra.

Una vez identificados los puntos de los elementos de captación, se procedió a identificar la(s) microcuenca(s) que tienen efecto en cada tramo o cruce de calle, para así poder sumar los gastos Q (caudal) que tienen influencia en cada tramo de la red de tuberías del sistema de alcantarillado propuesto funcionando a gravedad aprovechando la conformación topográfica actual de las calles; y en seguida se procedió con los cálculos del diámetro del tubo con sus características, considerando en cada intersección de calles un pozo de visita para la conexión entre tramos y que sirviese también con fines de mantenimiento.

El diámetro mínimo que se recomienda para las atarjeas en alcantarillado pluvial es de $30 \mathrm{~cm}$ con objeto de evitar frecuentes obstrucciones en las tuberías abatiendo por consiguiente los costos de conservación y operación del sistema. En cuanto a las pendientes de la tubería, ésta debe ser lo más semejante posible, a las del terreno natural con objeto de tener excavaciones mínimas, pero 
tomando en cuenta que la pendiente de la tubería deberá ajustarse en caso de que la velocidad alcanzada sea mayor a la permisible, manejando entonces una pendiente mínima de 5 al millar (CONAGUA, 2007). Para el cálculo del diámetro de la tubería en la primera parte del sistema de conducción (red de tuberías) se parte de la ecuación general de Manning presentada en forma del diámetro (Ec. 5) considerando en su diseño un tirante máximo del agua en el tubo del $81 \%$ del diámetro respectivo en cada tramo, y tomando en cuenta material de PVC para el tubo respectivo.

La ecuación de Manning normalmente es presentada en términos de la velocidad de flujo del agua (Gribbin, 2017).

$$
\mathrm{D}=\left[\frac{3.208 Q n}{s^{1 / 2}}\right]^{3 / 8}
$$

Siendo:

$\mathrm{D}=$ Diámetro del tubo $(\mathrm{m})$

$\mathrm{Q}=$ Gasto o caudal de agua en el tramo $\left(\mathrm{m}^{3} / \mathrm{s}\right)$

$\mathrm{n}=$ Coeficiente de Manning según el material del

tubo (adimensional)

$\mathrm{S}=$ Pendiente en el tramo de calle $(\mathrm{m} / \mathrm{m})$

\subsubsection{CAPTACIÓN DEL AGUA.}

El agua pluvial que escurre por las calles del campus universitario se propuso captarla de dos formas distintas. La primera fue a través de una serie de bocas de tormenta distribuidas estratégicamente en la red, y cuyo propósito es captar el agua y enviarla hacia la tubería que pasas por la calle. Estos elementos son las estructuras que recolectan el agua que escurre sobre la superficie del terreno, se ubican principalmente aguas arriba del cruce de calles, o bien aguas debajo de los cruces según el flujo del agua según el agua a captar en el tramo.

Las bocas de tormenta, también suelen colocarse en puntos bajos de terreno evitando la acumulación de agua, generalmente poseen una rejilla que permite el acceso del agua y bloquea el paso de residuos de tamaños mayores que pueden obstruir las obras de conducción; están constituidas por una caja receptora bajo la rejilla que funciona como desarenador donde se depositan las materias que son arrastradas por el escurrimiento del agua (IMTA, 2013). Existen varios tipos de bocas de tormenta o coladeras pluviales, de manera que de acuerdo a su diseño y ubicación, se clasifican en coladeras de: piso, banqueta, piso y banqueta, longitudinales de banqueta y transversales de piso (CONAGUA, 2007). De la caja receptora sale una tubería flexible de material PVC de descarga que se acopla e inserta directamente en el lomo del tubo colector en los tramos y puntos de colección del agua referidos a la red de tuberías del alcantarillado pluvial.

La capacidad (caudal Q) para una boca de tormenta o coladera pluvial se determinóutilizando principios de hidráulica; para el tipo de coladeras de banqueta se revisó su funcionamiento para dos casos posibles, uno fue cuando la estructura de dicha coladera trabaja como orificio, y el segundo caso es cuando predominaba el principio de vertedor de demasías, utilizando para ello las ecuaciones (6) y (7). Al considerar que funcionan hidráulicamente como vertederos para pequeñas alturas de agua (d) menor o igual a la altura de la guarnición (h) se aplica Ec. 6 (IMTA, 2013).

$$
Q=C P d^{1.5}
$$

Donde:

$\mathrm{Q}$ = capacidad o gasto recolectado por la coladera, $\left(\mathrm{m}^{3} / \mathrm{seg}\right)$.

$C=$ Coeficiente de descarga con valor sugerido $=1.25$

$\mathrm{P}=$ es equivalente al perímetro sin contar el lado de la cuneta $=(\mathrm{L}+1.8 \mathrm{~W})$

$L=$ longitud de la abertura, $(\mathrm{m})$.

$\mathrm{W}=$ Ancho de la coladera, $(\mathrm{m})$.

$d=$ La altura de la coladera medida desde la base, hasta la altura libre del agua (m)

En otra situación al considerar que la boca de tormenta funciona como un orificio significa que se presentan alturas del agua (d) en la cuneta mayores que la altura del orificio ubicado bajo la banqueta $(h)$; por lo que en términos prácticos la capacidad (Q) de la coladera se obtuvo mediante Ec. 7 (CONAGUA, 2007). En este caso el agua acumulada en la cuneta de la calle resultaba superior ahogando el orificio bajo la banqueta, incluso dicho nivel del agua en la cuneta supera la altura de la guarnición:

$$
Q=\operatorname{CoA} \sqrt{2} \operatorname{gd}
$$

Siendo:

$\mathrm{Q}$ = caudal recolectado por la coladera o rejilla, $\left(\mathrm{m}^{3} / \mathrm{seg}\right)$

$\mathrm{Co}=$ coeficiente de orificios, con valor sugerido de 0.60 
$\mathrm{A}$ = área del orificio abierto en la guarnición, $\left(\mathrm{m}^{2}\right)$

$\mathrm{g}=$ aceleración de la gravedad $\left(9.81 \mathrm{~m} / \mathrm{s}^{2}\right)$

$\mathrm{d}=$ tirante del agua sobre la coladera, $(\mathrm{m})$

La boca de tormenta del tipo combinada (de banqueta y piso), para este caso específico el caudal (Q) se determinó considerando la suma de los caudales obtenidos por separado utilizando las ecuaciones 6 y 7 descritas anteriormente.

La segunda forma de captación de agua de lluvia fue a través de tanques cisterna. Esta propuesta consistió en ubicar tanques (cárcamos) enterrados para almacenar la mayor parte del agua que aporta la microcuenca interna 6 (Ci6), en donde el agua escurrida se concentra sobre las areas de esparcimiento de los departamentos de Geología, Fisica, Polímeros y áreas adyacentes, integrándose finalmente el agua sobrante sobre la calle Sabiduría en el campus, con rumbo hacia la avenida Colosio de la vialidad urbana en la ciudad.

\subsubsection{APROVECHAMIENTO SOSTENIBLE DEL AGUA PLUVIAL.}

Desde la última década ha sido creciente el interés en la gestión integrada del agua urbana y la idea de que las aguas pluviales urbanas podrían proporcionar un valioso recurso de agua; en general, este concepto se ocupa de posibilitar una gestión más sostenible de los ambientes pluviales urbanos (Brown R., 2005). La necesidad de afrontar la gestión de las aguas pluviales desde una perspectiva diferente a la convencional, que combine aspectos hidrológicos, medioambientales y sociales, está llevando a un rápido aumento a nivel mundial del uso de Sistemas Urbanos de Drenaje Sostenible (SUDS), también conocidos como BMP's (Best Management Practices) o WSUD (Water Sensitive Urban Design), entre otras acepciones (Perales-Momparler y Doménech, s.f.).

En ese sentido, como tercera etapa del presente estudio, se enfocó a integrar el caudal total de agua pluvial del sistema propuesto, el cual se recolecta en el pozo de visita de la última intersección de calles del trazo marcado en la discretizaciòn del sistema pluvial (Ave. Universidad y calle del Conocimiento) identificado con el pozo No. 5 de donde el agua sigue su flujo hacia el pozo final No. 6 ubicado al finalizar la calle Universidad a escasos 25 metros del cruce con la avenida De la Reforma de la vialidad urbana en la ciudad (Fig. 7). De este pozo de visita final se propuso desviar el total de agua total hacia una caja derivadora, donde dicha caja primero recibe el caudal total, y después lo deriva hacia un canal de tierra de sección trapecial, en el cual se genera un flujo de agua a superficie libre a gravedad y a su vez, se induce la infiltración del agua a través del mismo canal y por un sistema de pozos de infiltración en serie que se conectan al canal, mismos que toman el agua del canal para filtrarlo al subsuelo, y generar una recarga del agua subterránea en esa zona.

El gasto total mencionado es el que puede ocurrir en un periodo de retorno $\operatorname{Tr}=10$ años derivado de las microcuencas siguientes: Ce1, Ce2, Ci2, Ci1, Ci5, y 50\% de (Ci4 y Ce3). El diseño del canal se basó en la ecuación universal de Manning; la cual se expresa para flujo uniforme en función de la velocidad media del agua (Gribbin, 2017), como se muestra:

$$
V=\frac{1}{n} R^{2 / 3} S^{1 / 2}
$$

Donde:

$\mathrm{V}=$ velocidad media de flujo en el canal, ( $\mathrm{m} / \mathrm{seg}$.)

$\mathrm{R}=$ radio hidráulico, $\mathrm{R}=\mathrm{A} / \mathrm{P}_{\mathrm{m}},(\mathrm{m})$

$\mathrm{A}=$ área hidráulica de la sección trapecial del canal, $\mathrm{A}=\mathrm{bd}+\mathrm{zd}^{2}\left(\mathrm{~m}^{2}\right)$

$\mathrm{P}_{\mathrm{m}}=$ perímetro mojado, $\mathrm{Pm}=\mathrm{b}+2 \mathrm{~d} \sqrt{1+Z^{2}}(\mathrm{~m})$ $\mathrm{n}=$ rugosidad del canal; $\mathrm{n}$ de Manning para el canal de tierra (adimensional)

$\mathrm{S}=$ pendiente de la plantilla del canal, $(\mathrm{m} / \mathrm{m}$.)

$\mathrm{Z}=$ es el talud del canal de tierra. $\mathrm{Z}=1.5$

Los parámetros hidráulicos que permiten iniciar el proceso de diseño del canal fueron: área hidráulica, perímetro mojado y radio hidráulico, mismos que para el canal de sección transversal trapezoidal son obtenidas trigonométricamente. Al sustituir el gasto $(\mathrm{Q})$ en la ecuación de manning y considerando que $\mathrm{Q}=\mathrm{AV}$ por principios básicos de hidráulica, y dejando la ecuación en la primera parte con términos conocidos $(\mathrm{Q}, \mathrm{n}$ y $\mathrm{S})$ y los términos que son incógnita (A y R) sección derecha de la ecuación, según la ecuación (9):

$$
\frac{Q \mathbf{n}}{\sqrt{\mathbf{S}}}=\mathbf{A} \mathbf{R}^{2 / 3}
$$

La ecuación vista de esa manera representa la solución de máxima eficiencia del canal, el proceso implica que el diseño tendrá una solución 
que arroje valores que cumplan la igualdad de la ecuación (9) mostrada; por lo que, el proceso es iterativo ya que se proponen valores de la geometría del canal: ancho y tirante (b y d) hasta lograr el cumplimiento de la ecuación (9). La sección obtenida en su diseño debe cumplir que el flujo de agua en el canal debe presentarse con una velocidad adecuada, ya que valores muy bajos pueden impedir que el agua fluya adecuadamente, mientras que valores altos de la velocidad del agua pueden provocar daño en la plantilla y taludes del canal, por lo que el tipo de flujo deseable en el canal es un flujo lento, el cual se le denomina también flujo subcritico.

La teoría de canales para flujo uniforme identifica tres tipos de flujo en un canal relacionados con su diseño y de acuerdo a la energía con la cual se conduzca el agua, siendo estos el flujo crítico, subcritico y supercrítico. El nivel de energía del agua en el canal se refiere al valor de la suma de la energía de posición, de presión y de velocidad para un gasto Q de flujo constante; asi entonces el tipo de flujo deseable es el subcritico (CONAGU, 2007).

Por otra parte, la escorrentía del agua pluvial en general en las cuencas urbanas debe ser canalizada con enfoques más sustentables para lograr un mejor aprovechamiento y a su vez, disminuir la problemática de las inundaciones y los riesgos de daños a la infraestructura. Una forma de lograr lo anterior es disminuyendo la escorrentía de agua y caudales máximos que mediante estructuras de retención y desplazando el agua a sitios menos impermeables.

En ese sentido, se propuso un proceso de infiltración del agua pluvial al subsuelo, mediante un sistema en serie de pozos de infiltración, los cuales son elementos hidráulicos que consisten en excavaciones cilíndricas de profundidad somera, que estarán rellenas de material granular (boleos, y gravas) que permiten infiltrar el agua de lluvia que colecta la caja derivadora directamente al suelo en espacios reducidos en la longitud del canal de tierra propuesto. Esta técnica tiene la ventaja de poder ser aplicada en zonas en las cuales el estrato superior de suelo es poco permeable, como es el caso de zonas altamente urbanizadas.

\begin{tabular}{|c|c|c|c|c|}
\hline Cuenca & Coeficiente C & $\mathbf{I}(\mathbf{m m} / \mathbf{h r})$ & $\mathbf{A}\left(\mathbf{k m}^{2}\right)$ & $\mathbf{Q}\left(\mathbf{m}^{\mathbf{3}} / \mathbf{s}\right)$ \\
\hline $\mathrm{Ce} 1$ & 0.706 & 87.539 & 0.08211 & 1.409 \\
\hline
\end{tabular}

\section{RESULTADOS Y DISCUSIÓN.}

Al procesar la información de precipitaciones máximas diarias, se obtuvo la relación de lluvias, máximas diarias en 24 horas para diversas duraciones de tormenta y periodos de retorno, representadas en las curvas P-d-Tr, es la mostrada en la Figura 5, siguiente:

Figura 5. Curvas de precipitación, duración y periodo de retorno: CURVAS P-d-Tr

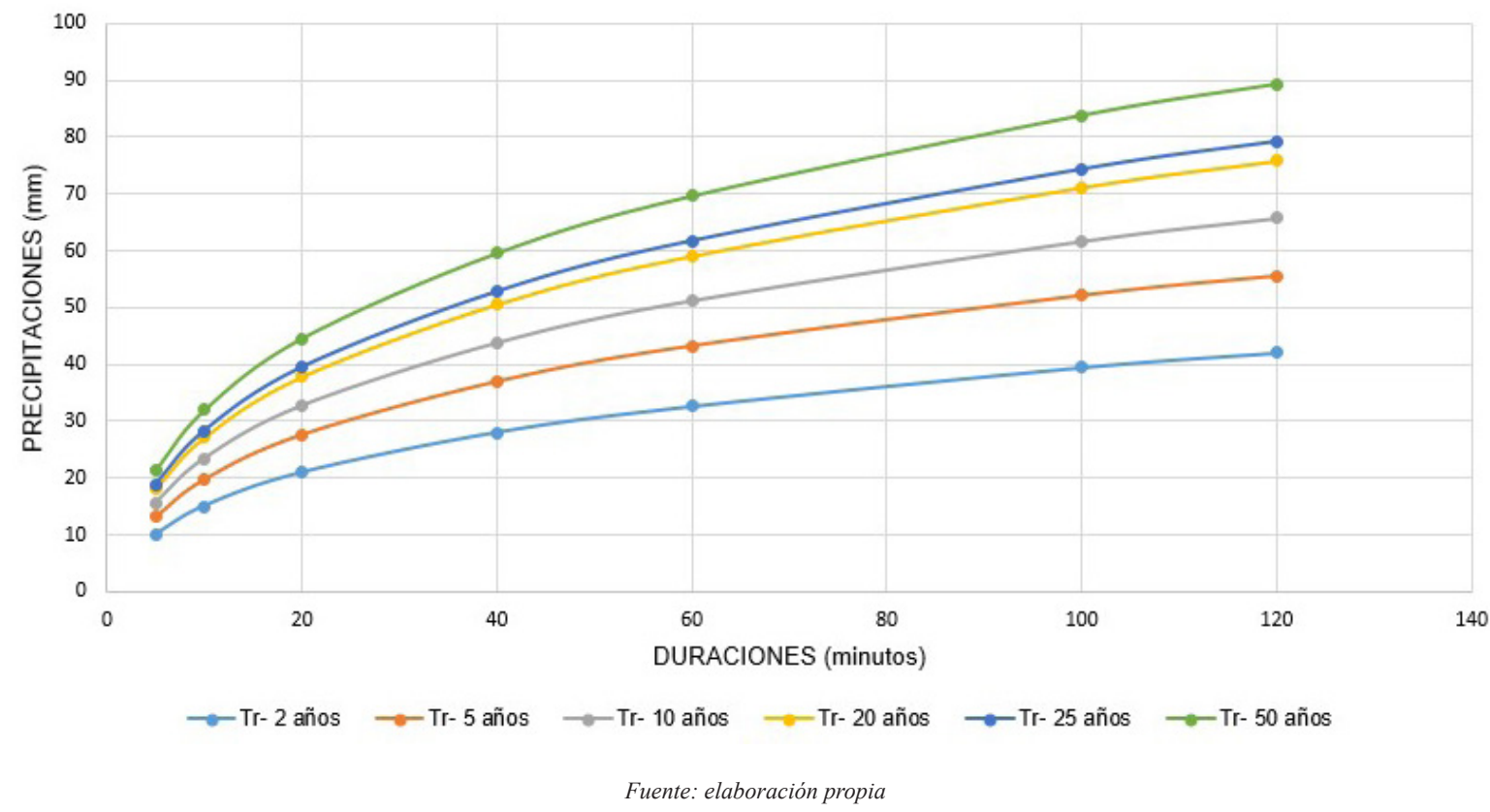


Cualquier valor de precipitación $(\mathrm{mm})$ para un periodo de retorno considerado en la gráfica resultante, puede transformarse en intensidad de lluvia $(\mathrm{mm} / \mathrm{hr})$ al dividir el valor de la precipitación entre la duración de la tormenta.

El caudal de agua (Q) que resultó a través de la aplicación integra del método racional, considerando la duración de tormenta mediante como el tiempo de concentración promedio y para un $\mathrm{Tr}=10$ años, asi como el coeficiente de escurrimiento en cada microcuenca del campus universitario es el que se muestra en la Tabla 2.

Por otro lado, el resultado que arrojó el diseño de la red de tuberías del sistema de drenaje pluvial con funcionamiento a gravedad, considera un flujo uniforme del agua y un tirante normal (altura) del agua (Yn) en el tubo trabajando al $81 \%$ del diámetro, así como la velocidad del agua en cada uno de los tramos de la red, se muestran en la Tabla 3. Los valores de la velocidad son satisfactorios ya que están en el rango deseable (3 $\mathrm{m} / \mathrm{seg}$. a $5 \mathrm{~m} / \mathrm{seg}$.).

Respecto al sistema de captación del agua pluvial resultaron varios tipos de bocas de tormenta, los cuales, de acuerdo a su diseño y ubicación en las calles, resultaron tres casos: del tipo transversal, de banqueta, y combinada. El mayor caudal que ingresa al campus universitario es generado en la cuenca externa $1(\mathrm{Ce} 1)$, cuya descarga resultó de $\mathrm{Q}=1.4 \mathrm{~m}^{3} / \mathrm{seg}$. Para captar el $50 \%$ de este caudal se propuso una rejilla transversal tipo Irving de longitud $\mathrm{L}=2.20 \mathrm{~m}$, y ancho $\mathrm{b}=0.86 \mathrm{~m}$., con un área de aberturas $\mathrm{A}=1.064 \mathrm{~m}^{2}$, misma que se conectará al Pozo P1 donde inicia la red de tuberías en la Av. Universidad.

Tabla 2. Gasto máximo para período $\operatorname{Tr}$ de 10 años

Fuente: elaboración propia

\begin{tabular}{|c|c|c|c|c|}
\hline $\mathrm{Ce} 2$ & 0.81 & 100.929 & 0.00378 & 0.0858 \\
\hline $\mathrm{Ce} 3$ & 0.81 & 116.223 & 0.00409 & 0.1070 \\
\hline $\mathrm{Ci} 1$ & 0.549 & 75.332 & 0.02861 & 0.3287 \\
\hline $\mathrm{C}$ 2 & 0.64 & 86.210 & 0.01106 & 0.1696 \\
\hline $\mathrm{Ci3}$ & 0.732 & 74.727 & 0.01989 & 0.3023 \\
\hline $\mathrm{Ci4}$ & 0.6915 & 74.420 & 0.05888 & 0.8418 \\
\hline $\mathrm{Ci5}$ & 0.721 & 114.885 & 0.01379 & 0.3174 \\
\hline $\mathrm{Ci6}$ & 0.5371 & 87.809 & 0.09611 & 1.2592 \\
\hline $\mathrm{Ci} 7$ & 0.51 & 71.381 & 0.04058 & 0.4101 \\
\hline $\mathrm{Ci} 8$ & 0.484 & 98.712 & 0.04372 & 0.5803 \\
\hline $\mathrm{Ci9}$ & 0.4947 & 102.927 & 0.08166 & 1.155 \\
\hline
\end{tabular}

Tabla 3. Diámetro de la tubería y velocidad media de flujo

Fuente: elaboración propia

\begin{tabular}{|c|c|c|c|c|c|}
\hline Tramo & $\begin{array}{c}\text { Caudal Q } \\
\left(\mathbf{m}^{3} / \mathbf{s}\right)\end{array}$ & $\begin{array}{c}\text { Diámetro } \\
\text { comercial } \mathbf{( m )}\end{array}$ & $\begin{array}{c}\text { Tirante } \\
\mathbf{Y n}(\mathbf{m})\end{array}$ & $\mathbf{R h}(\mathbf{m})$ & $\begin{array}{c}\text { Velocidad } \\
(\mathbf{m} / \mathbf{s})\end{array}$ \\
\hline P1-P5 & 1.409 & 0.91 & 0.7371 & 0.2769 & 3.66 \\
\hline P2-P3 & 0.558 & 0.61 & 0.4941 & 0.1856 & 3.62 \\
\hline P3-P4 & 0.949 & 0.91 & 0.7371 & 0.2769 & 3.81 \\
\hline P4-P6 & 0.317 & 1.22 & 0.9882 & 0.3712 & 4.97 \\
\hline P6- Caja D. & 0.317 & 1.22 & 0.9882 & 0.3712 & 4.97 \\
\hline P5-P4 & 0.329 & 0.91 & 0.7371 & 0.2769 & 4.95 \\
\hline
\end{tabular}


El caudal de la microcuenca interna Ci6 resultó de $1.25 \mathrm{~m}^{3} / \mathrm{seg}$, donde parte de este escurrimiento de agua se queda atrapado en las areas verdes de los departamentos de Geología, Fisica y en areas adyacentes. Por lo que, el aprovechamiento del agua en este caso, se propuso captarla mediante cuatro tanques cisternas de $90 \mathrm{M}^{3}$ de capacidad con dimensiones de $10 \mathrm{~m} \times 3 \mathrm{~m} \times 3 \mathrm{~m}$. La figura 6 ilustra los elementos resultantes de captación.

El caudal total captado en el sistema de red de tuberías en su último tramo por la avenida universidad descarga en el pozo de visita 6 (P6), del cual se envía hacia el sitio de aprovechamiento sustentable del agua pluvial que inicia en la caja derivadora (Fig. 7). Dicho caudal de agua que puede ocurrir en un periodo de retorno $\operatorname{Tr}=10$ años derivado de las microcuencas siguientes: $\mathrm{Ce} 1, \mathrm{Ce} 2$, $\mathrm{Ci} 2$, $\mathrm{Ci} 1, \mathrm{Ci} 5$, y $50 \%$ de (Ci4 y Ce3) y suma un total de $3.0 \mathrm{~m}^{3} / \mathrm{seg}$. Siendo que este caudal recolectado se descarga a un canal de tierra de sección trapecial en una longitud aproximada de 190 metros, y en cuyo recorrido el agua es infiltrada de manera natural al subsuelo a través del mismo canal y de los pozos de infiltración.

Figura 7. Desvío del caudal de agua pluvial hacia la caja derivadora

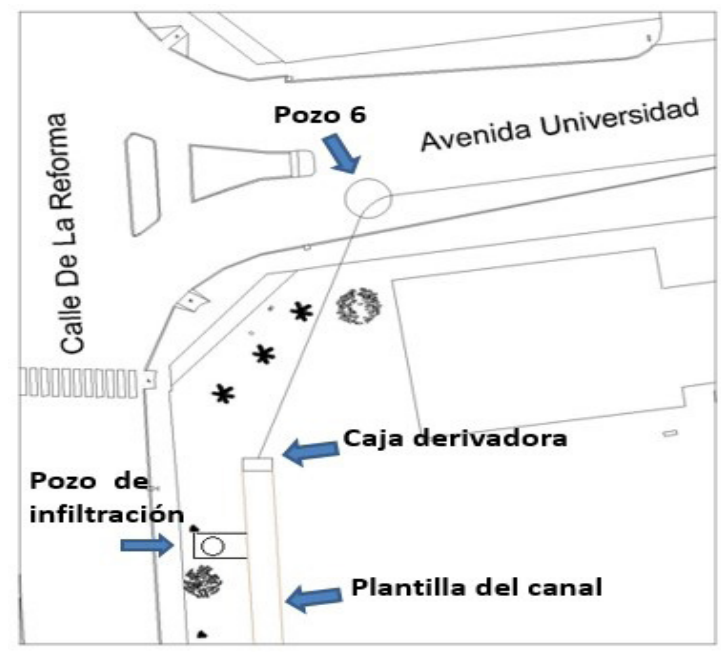

Figura 6. Rejillas, bocas de tormenta y cisternas en las vialidades del Campus

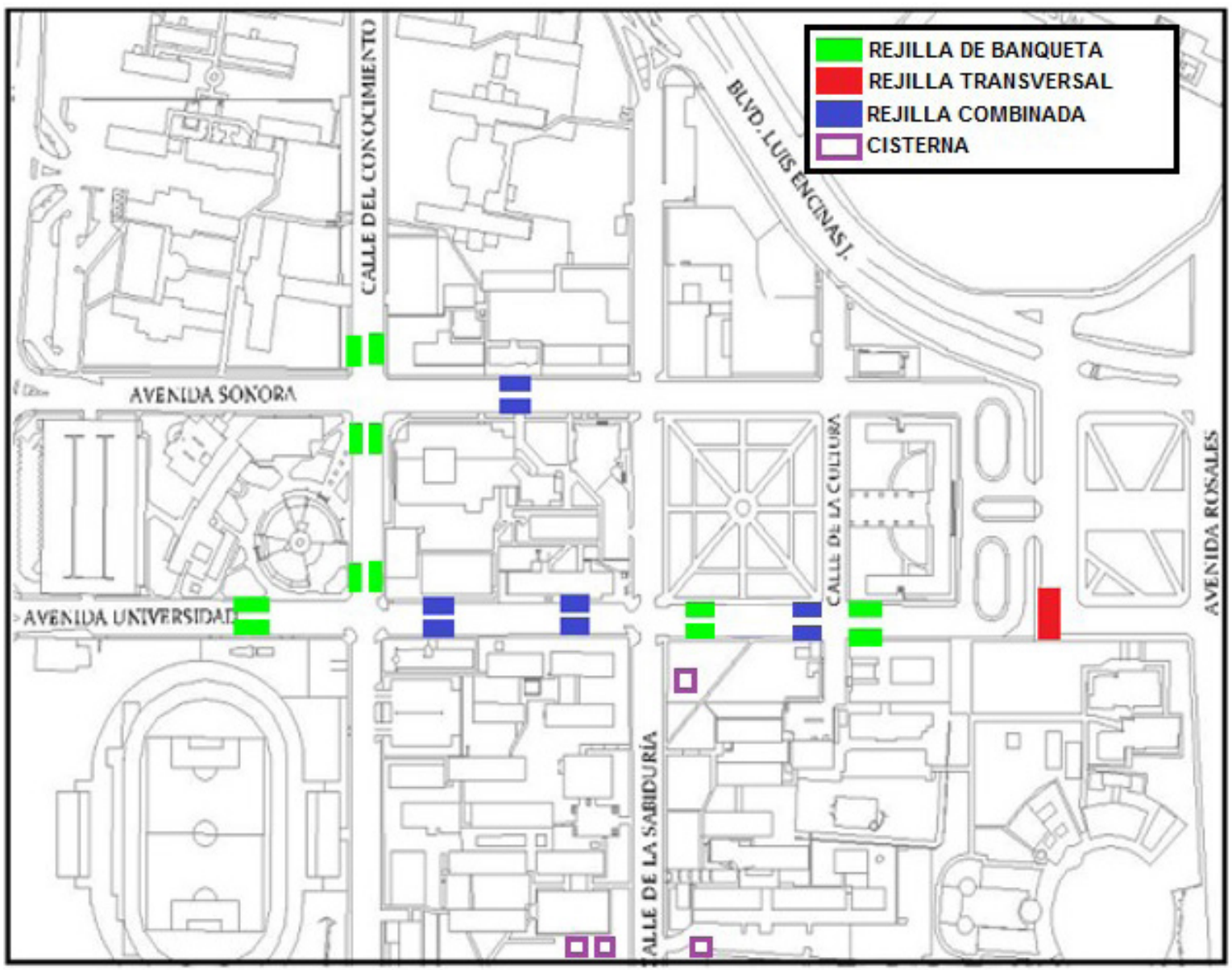

Fuente 6 y 7: elaboración propia 
El canal de tierra de secciòn trapecial resultò con un ancho de plantilla de 1.0 metros, y un tirante de agua de $0.93 \mathrm{~m}$., con un bordo lbre de $0.15 \mathrm{~m}$., y un ancho total de la superifcie libre del agua de $3.8 \mathrm{~m}$. (fig. 8). De alguna manera, esto guarda cierta similitud con el plan de aprovechamiento sustentable de la Universidad de Victoria, Australia, el cual consideró entre otros, la adecuación de estacionamientos en areas permeables, y canales de tierra trapeciales filtrantes con una ancho de plantilla de 0.6 a 2.4 mts., y pozo estanque de infiltración del agua al subsuelo (RCL, 2004).

Figura 8. Canal de tierra (arcilla) excavado en el sitio

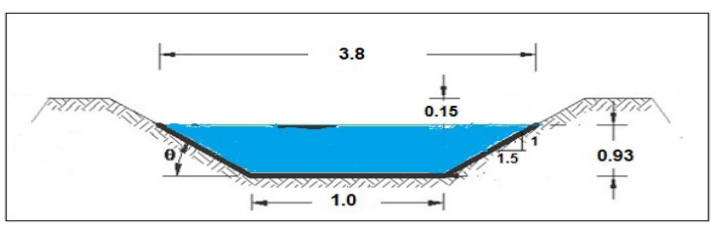

Fuente: elaboración propia

El canal propuesto recibirà el agua que descarga la caja derivadora de secciòn cuadrada de $2.5 \times 2.5$ $\mathrm{m}$. y altura $\mathrm{h}=3.8 \mathrm{~m}$; Mientras que el canal como elemento de partida toma el agua recolectada en la caja derivadora mediante el tubo que descarga en una pequeña zanja provista de materiales pétreos (arena, grava y boleo).

El flujo del agua en el canal resultò con una velocidad media de $1.3 \mathrm{~m} / \mathrm{seg}$, siendo un flujo lento y adecuado. Lo cual es aceptable ya que el valor obtenido para el tirante critico (dc) resultó $\mathrm{dc}=0.70 \mathrm{~m}$. cumpliéndose la condición $\mathrm{dc}<\mathrm{d}$ garantizando asi un flujo subcritico (lento) del agua en el canal de tierra, lo cual es ideal para que en la conducciòn del agua se favorezca la infiltracion al subsuelo mediante el mismo cnal, asi como el tramo corto de zanja y los pozos de infiltracion propuestos a cada 20 metros en el eje del canal (Fig. 9).

La estratigrafia del terreno ha sido detrminada por el laboratorio de Geotecnia del Dpto. de Ing. Civil y Minas, de la Universidad de Sonora, de acuerdo a la comunicación personal del Ing. Oscar R. Rodríguez indicando que el material filtrante en el sitio inicia a una profundidad de tres metros con una permeabilidad (K) del estrato de grava 150 $\mathrm{m} /$ día $\left(1.7 \times 10^{-3} \mathrm{~m} / \mathrm{s}\right)$. Por lo que, se propuso una profundidad ligeramente mayor de 3.5 metros para
Figura 9. Aprovechamiento sustentable del agua pluvial (canal y pozos de infiltración)
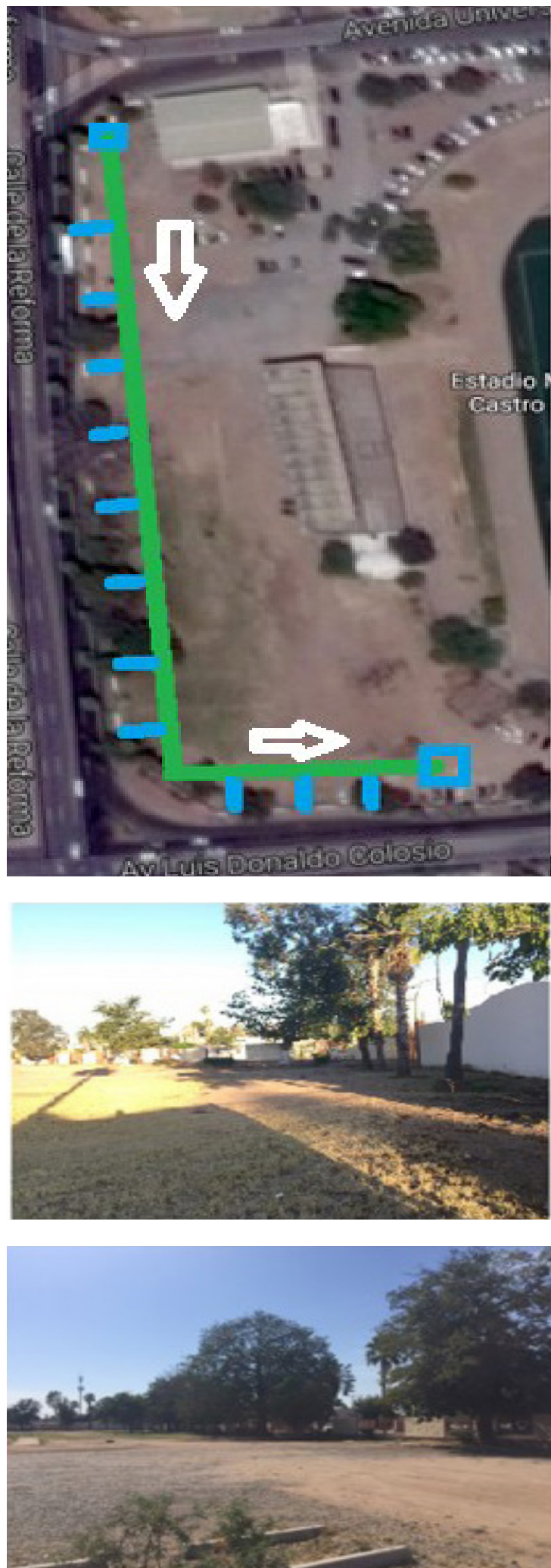

Fuente: elaboración propia 
cada uno de los pozos de infiltracion en serie de forma cilindrica con un diàmetro de $1.0 \mathrm{~m}$. (Fig. 10). Fue necesario proponer una segunda caja del agua pluvial al finalizar el eje del canal, la cual su función es una obra de demasías (excedencias), cuyo propósito es enviar el agua pluvial sobrante hacia afuera del campus por la calle Colosio que se localiza a escasos 3 metros frente a dicha caja después del muro perimetral del campus universitario.

Fig. 10 Elementos en Isométrico: canal, zanja y pozo de infiltración.

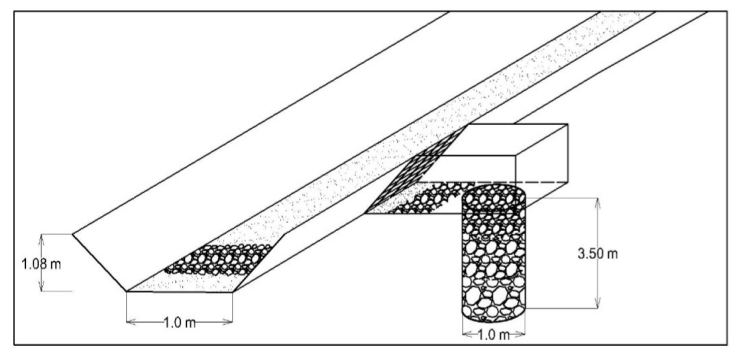

Fuente: elaboración propia

En esta temática, Gonçalves y otros (2016) abordaron un estudio para la mejora del paisaje en un proceso del planeamiento del campus en la universidad de São Carlos, Brasil, tales estudios dieron como resultado proyectos paisajísticos que consistieron dos áreas amplias que sirvieran para la Infiltración del agua pluvial, y adicionalmente un pozo de infiltración de agua hacia al subsuelo; con ello lograron confirmar la necesidad del reconocimiento de las técnicas compensatorias por la comunidad usuaria para fines de aceptación e identificación con los espacios, proporcionando la gestión integrada de infraestructura y creando un paisaje sustentable. De igual manera, Torres y otros (2012), señalan que el estudio de aprovechamiento de agua de lluvia para el Campus de la Universidad Javeriana en Bogotá, fue mediante análisis a través de microcuencas, para proponer construir diferentes tanques y redes que lograran ser operadas por sistemas a gravedad sin afectar los espacios del campus.

\section{CONCLUSIONES.}

Para abordar el problema del escurrimiento de agua pluvial en el campus Hermosillo de la Universidad de Sonora fue necesario dividir la superficie de estudio en un conjunto de microcuencas. De ahí que se identificaron un total de 12 microcuencas, de las cuales se denominaron tres externas al campus (Ce), y nueve microcuencas internas (Ci); de estas la microcuenca que generaron el mayor caudal es la Ce1 y la Ce2.

En el caso de las tormentas que se presentan en zonas cálidas, estas suelen ser del tipo torrencial, pues son lluvias intensas y de corta duración, los resultados arrojaron que la máxima duración fue de 33 minutos, y a su vez, la precipitación máxima ocurrida fue de $40 \mathrm{~mm}$ en la microcuenca Ci7, lo que representa una intensidad de lluvia de $71.3 \mathrm{~mm} / \mathrm{hr}$., diferente es cuando la duración en la microcuenca $\mathrm{Ce} 3$ fue de 12.9 minutos, y una precipitación de $25 \mathrm{~mm}$, genera una intensidad de lluvia de $116.2 \mathrm{~mm} / \mathrm{hr}$. Esta situación provoca escurrimientos agresivos por el agua, y provoca inundación cuando el agua es retenida por infraestructura que impide el paso del fluido, como es el presente caso.

Se propone una metodología con aplicaciones teórico practica para crear un sistema de drenaje pluvial que no tiene precedente alguno, la propuesta es de dos fases generales, primero de captar el agua pluvial mediante una red de tuberías de material PVC enterrada para evitar el conflicto al interior del campus, y la segunda mediante el aprovechamiento sustentable de esa misma agua captada; lo cual deberá ser dirigida hacia una caja receptora y derivadora para luego conducirla y pasearla por un canal de tierra para generar una infiltración del agua al subsuelo.

Se establecen las bases para lograr un cuidado al medio ambiente, ya que la infiltración del agua pluvial a través de la serie de pozos de infiltración propuestos es factible, puesto que la posición del nivel de agua subterránea es mucho más profunda que los 3.5 metros de profundidad propuesta para dichos pozos; convirtiendo esto en una recarga potencial de agua en temporada de lluvias para la zona acuífera urbana. Esto favorece la disminución del escurrimiento del agua pluvial en la zona urbana aledaña a la Universidad, beneficiándose la ciudad por la disminución del volumen de agua de escorrentía en la parte urbana que le corresponde a este sector. Mediante esta alternativa generada por el estudio desarrollado, se presenta una area de oportunidad para la Universidad de Sonora en esta temática y se debe evitar daños a la comunidad universitaria y a la infraestructura, lo cual se puede traducir a un cuidado del medio ambiente.

El caudal de agua que en su caso, pudiera exceder 
en el sistema propuesto cuando el periodo de retorno elegido fuese rebasado, el agua excedente finalmente puede reintegrarse al escurrimiento urbano, ya sea a través de la descarga que podría generase de flujo excedente en la calle De la Sabiduría que fluye hacia la zona sur del campus con dirección a la avenida Colosio, y la posibilidad de que la caja prevista de demasías (ubicada al final del canal) también haga lo mismo, previamente se haya captado el agua en los tanques cisterna propuestos para almacenar temporalmente el agua, y darle uso futuro en el riego de areas verdes.

Por otro lado, la situación de agua excedente también puede ser infiltrada en la superficie de terreno adyacente al eje del canal, ya que es un sitio utilizado como estacionamiento vehicular que utilizan como complemento los universitarios; ya que puede ser preparada la superficie con material granular filtrante. Además de plantar árboles en el sector perimetral que genere una barrera verde y logren coadyuvar en el amortiguamiento del ruido urbano.

La autoridad universitaria en turno merece considerar en su nuevo Plan de Desarrollo Institucional (PDI) un plan integral para la gestión sostenible del agua de lluvia, en el que se incluya las metas y acciones específicas, para cada uno de los campus; en principio para el campus Hermosillo que es el más importante y de mayor magnitud en todos los sentidos. C

\section{REFERENCIAS BIBLIOGRÁFICAS.}

Benavides, G., Perales-Momparler, S. (2008). Integración de las aguas pluviales en el paisaje urbano: un valor social a fomentar. I Congreso Nacional de Urbanismo y Ordenación del Territorio. Bilbao. http:// docplayer.es/15350691-Integracion-delas-aguas-pluviales-en-el-paisaje-urbanoun-valor-social-a-fomentar.html, [20 mayo 2017].

Brown, R. (2005).'Impediments to Integrated Urban Stormwater Management: The Need for Institutional Reform". Journal Environmental Management. Vol.36 (3), pp. 455-468.

Brown, R., Keath, N., Wong, T.H.F. (2009). "Urban water management in cities: historical, current and future regimes". Water Science and Technology 59 (5), pp. 847- 855.

Bryan E.; Lundy L. (2016). "Implementing sustainable drainage systems for urban surface water management within the regulatory framework in England and Wales". Journal of Environmental Management, vol. 183, (3), pp. 630-636.

Campos-Aranda, D. F. (1998). Procesos del ciclo hidrológico. S.L. P. Mex. Ed. UASLP.

Campos-Aranda, D.F. (2010). Introducción a la hidrología urbana. San Luis Potosí, México.

ComisiónNacional delAgua. CONAGUA. (2007). Manual de agua potable, alcantarillado y saneamiento. Alcantarillado Pluvial. ftp:// ftp.conagua.gob.mx/Mapas/libros\%20 pdf $\% 202007 /$ Datos $\% 20 B \%$ E1 sicos.pdf, [6 mayo 2017].

Comisión Nacional del Agua. CONAGUA. (2016). Servicio Meteorológico Nacional. http://smn.cna.gob.mx/es/ [19 Octubre 2016].

Chi-Feng, Chen., Chung-Ming, Liu. (2014). "The definition of urban stormwater tolerance threshold and its conceptual estimation: an example from Taiwan". Natural Hazards, Vol. 73, (2), pp. 173-190.

Gonçalves, L.; Barbassa, A.; Pereira, T. (2016). El Proyecto Paisajístico como elemento integrador e identificador de técnicas compensatorias de drenaje. I Congreso Internacional Towards Green Cities. Mérida, México.

Gribbin, J. (2017). Introducción a la Hidráulica e Hidrología con aplicaciones para la administración del agua pluvial. 4a . Edición, Ed. Cengage Learning. ISBN 978-607-526003-7.

Instituto Mexicano de Tecnología del Agua, IMTA. (2013). Análisis y diseño numérico y experimental de bocas de tormenta para el desalojo de aguas pluviales.http://repositorio. imta.mx/handle/20.500.12013/735, [ $1^{\circ}$ junio 2017].

Instituto Nacional de Estadística, Geografía e Informática (INEGI). (2010). Censo de población y vivienda 2010. http://www. inegi.org.mx/est/contenidos/proyectos/

ccpv/cpv2010/Default.aspx [08 Enero 2016].

Katsifarakis, K., Vafeiadis, M., Theodossiou, N. (2015). "Sustainable Drainage and Urban Landscape Upgrading Using rain gardens. Site Selection in Thessaloniki, Greece". Agriculture and agricultural Science Procedia, (4), pp 338-347. 
Li Fei; Huan Feng; Yan H.; Tao T. (2015). "MultiObjective Optimal Design of Detention Tanks in the Urban Stormwater Drainage system: Framework Development and Case Study”. Water Resources Management, 29, (7), pp. 2125-2137.

Ojeda, A.; Alvarez-Chávez C.R.; Ramos, M.; Soto, F. (2017). "Determinants of domestic water consumption in Hermosillo, Sonora". Journal of Cleaner Production, 142, pp. 1901-1910.

Ojeda, A., Narváez, A., Quintana, J. (2014). “Gestión del agua doméstica urbana en Hermosillo, Sonora". Cuadernos de Geografia 23 (1), pp. 147-164.

Perales-Momparler S., Doménech, I. (s.f.). "Los Sistemas Urbanos de Drenaje Sostenible: Una Alternativa a la Gestión del Agua de Lluvia".

http://ovacen.com/wp-content/uploads/2015/05/ gestion-del-agua-en-el-planeamiento.pdf, [30 abril 2017].

Perales-Momparler, S., Doménech, I., HernandezCrespo, C., Hernandez-Crespo, C., VallèsMoràn, F., Martin, M., Escuder-Bueno, I., Andreu, J. (2016). "The rol of monitoring Sustainable drainage systems for promoting transition Towards regenerative urban built environments: a case study in the Valencian region Spain". Journal of Cleaner Production, 163, pp. 5113-5124.

Poleto, C., Tassi, R. (2012). "Sustainable Urban Drainage Systems". En Muhammad Salik J. (eds). Chap. 3, en Drainage Systems, pp. 56-72. ISBN 978-953-51-0243-4.

RCL. (2004). Integrated Stormwater management plan. University of Victoria. Proyect No. 024367. https://www.uvic.ca/sustainability/ assets/docs/policy/integrated-stormwatermanagement-plan.pdf, [4 Agosto 2017].

Roy, A., Wenger, S., Fletcher, T., Walsh, Ch., Ladson, A., Shuster, W., Thurston, H., Brown, R. (2008). "Impediments and Solutions to Sustainable, Watershed-Scale Urban Stormwater Management: Lessons from Australia and the United States". Journal Environmental Management. 42, pp. 344-359.

Sharma, D., Kansal, A. (2013). "Sustainable City: A Case Study of Stormwater Management in Economically Developed Urban Catchments". Mechanism Design for Sustainability, pp. 243-263.
Sun, F., Yang, Z., Huang, Z. (2014). "Challenges and solutions of urban hydrology in Beijing". Water Resources Management, 28 (11), pp. 3377-3389.

Torres, A., Méndez-Fajardo, S., Lara-Borrero, J., Estupiñán, J., Zapata, H., Torres, O. (2012). "Hacia Equipamiento Urbano Sostenibles: Aprovechamiento de agua de lluvias en el Campus de la Pontificia Universidad Javeriana en Bogotá". Cuadernos de vivienda y Urbanismo, (5), 9, pp. 124-141.

Universidad de Sonora (2013). Plan de Desarrollo Institucional (PDI) 2013-2017. http:// www.uson.mx/institucional/pdi20132017.pdf, [30 mayo 2017].

Willems, P., Arnbjerg-Nielsen, K., Olsson, J., Nguyen; V.T.V. (2012). "Climate change impact assessment on urban rainfall extremes and urban drainage: Methods and shortcomings". Atmospheric Research. 103, pp. 106-118. 